\title{
Compósito nanoestructurado con propiedades superhidrofóbicas resistente a la corrosión mediante nanopartículas de caolín y polímeros reciclados
}

\author{
M. Valadez¹, J. González ${ }^{\star}$, E. Cantú1, B. Domínguez¹. \\ ${ }^{1}$ Universidad Tecnológica de Tecámac, División de Procesos Industriales, Área Nanotecnología, Carretera \\ Federal México-Pachuca Km 37.5, Col. Sierra Hermosa, Tecámac,55740, Estado de México. \\ m.antonio.valadez@hotmail.com, dominguezbrenda180@gmail.com, stephanus12co@gmail.com. \\ *Autor de correspondencia: gonzalezpalaciosjoseguillermo@gmail.com
}

\section{RESUMEN}

En la actualidad el estudio del fenómeno de la superhidrofóbicidad ha tenido avances muy significativos, uno de ellos asido la obtención de recubrimientos superhidrofóbicos a base de materiales compositos; con el fin de mejorar las propiedades de algunos materiales, como: inhibidores de corrosión, la reducción de la adherencia de líquidos en la superficie en estos materiales. Dado que algunos recubrimientos anticorrosivos son realmente costosos, incluyendo la manipulación de la técnica. Con la aplicación de la nanotecnología se ha tenido un mayor avance, esto es debido a los excelentes métodos para la obtención de recubrimiento con propiedades anticorrosivas y superhidrofóbicas. En el presente artículo, se presenta un composito superhidrofóbico a base de nano partículas de caolín-polímeros reciclados, con aplicación innovadora como un recubrimiento, presentando propiedades anticorrosivas y de no-mojabilidad; para aceros de bajo carbón, el composito se depositó con un robot cartesiano con deposición por spray, por ende, se obtuvieron recubrimientos homogéneos con características esperadas. La espectroscopia infrarroja por transformada de Fourier (FTIR), indica la presencia de los grupos funcionales ac. carboxílico (COOH) a 2920 y $2853 \mathrm{~cm}^{-1}$, característico de superficies altamente hidrofóbicas, además se aplicaron métodos de aproximación para efectuar la medición del ángulo de contacto (Reflexión y Aumento de imagen), los cuales demostraron que el ángulo es igual o se aproxima a $150^{\circ}$. Con ello se procedió a hacer ensayos de corrosión mediante cámaras que simulaban atmosferas corrosivas, se obtuvieron resultados favorables, el cual demuestra que el composito nanoestructurado posee las propiedades anticorrosivas y superhidrofóbicas.

Palabras Clave: Composito, Superhidrofóbico, Mojabilidad, Ángulo de contacto, Corrosión.

\section{INTRODUCCIÓN}

En los últimos años, el maravilloso fenómeno denominado como efecto de loto [1], ha sido base para grandes avances y aplicaciones, se caracterizan por tener un ángulo de contacto superior a $150^{\circ}$. Con ello vino el estudio de los materiales superhidrofóbicos que ha tenido un gran enfoque en la actualidad, esto debido a sus grandes propiedades que presentan, como lo son la repelencia al agua, la no mojabilidad y la anticorrosión [2]. Con grandes progresos significativos con la nanotecnología se han desarrollado técnicas accesibles, con el fin de obtener materiales superhidrofóbicos, sin embargo, algunos de ellos requieren un procesamiento complejo y con un elevado precio [3]. Es por eso que 
se han dedicado a buscar nuevas alternativas para obtener materiales con las mismas propiedades, con métodos más accesibles. Una de las grandes alternativas son la aplicación de materiales compositos superhidrofóbicos, que en la actualidad están activamente investigados, puesto que ofrecen una serie de ventajas en los materiales que sean depositados, es muy relevante mencionar su bajo costo y su síntesis no representa gran peligro hacia el medio ambiente. Con la nanotecnología se ha podido modificar químicamente su estructura con la ayuda de aditivos, con el fin de otórgale mayores propiedades contra los materiales ya existentes. En este artículo, se presenta una aplicación novedosa de composito como un recubrimiento superhidrofóbico, se logra mediante nanopartículas caolín $\left[\mathrm{Al}_{2} \mathrm{Si}_{2} \mathrm{O}_{5}(\mathrm{OH})_{4}\right]$ - polímeros reciclados, cabe mencionar que el caolín es un mineral natural, frecuentemente utilizado para reforzar recubrimientos [4], estudios actuales han desarrollado estrategias para mejorar las propiedades de anticorrosión esto mediante la experimentación de la introducción de materiales compositos, con el fin de reducir el daño que sufre de la superficie externa. Para la aplicación del recubrimiento se utilizó un robot cartesiano con deposición por spray, mencionar que fue desarrollado por el Dr. Serrano [5]. El uso de robots cartesianos es con la finalidad de hacer tareas dentro de la industria, como el posicionamiento de recubrimientos homogéneos, designados para tener una mayor exactitud para adaptarlo a las diferentes necesidades que vayan surgiendo.

\section{MARCO TEÓRICO}

La tecnología en la actualidad busca imitar las bondades que ofrece la naturaleza, en sus diferentes fenómenos; no hace mucho ya se discutía sobre un efecto que caracterizaba a la superficie de las flores de loto que tiene que ver con dos conceptos clásicos, capilaridad y mojabilidad [6]. Este asunto repercute más en la mojabilidad que se describe como la capacidad que tiene un líquido de extenderse o dejar una traza mínima o nula sobre el sólido que se extiende. La cuidadosa observación e investigación de estas características inusuales de los fenómenos encontrados en la naturaleza ha llevado a discutir las propiedades de no-mojabilidad en la hoja de loto, lo cual se ha convertido en un tema de interés en las últimas dos décadas. Debido al alto ángulo de contacto $\left(>150^{\circ}\right)$, las gotas de agua se desprenden fácilmente de la superficie de la hoja de loto y acumulan partículas de suciedad. En la actualidad el desarrollo y la aplicación práctica de los recubrimientos superhidrofóbicos, están siendo enfocados principalmente para que los procesos de fabricación no sean tan complejos y se obtengan estructuras de superficie más robustas además con mayor duración [7], Gracias a la nanotecnología se ha podido introducir la superhidrofóbicidad en las áreas como: de la construcción, la industria automotriz y la metalúrgica. A partir de ello, se han desarrollado grandes enfoques para la fabricación recubrimientos superhidrofóbicos a base de materiales compositos, generando superficies superhidrofóbicas con resistencia a la corrosión, debido a la composición del composito. Los materiales superhidrofóbicos actualmente están teniendo un mayor impacto, con ello se está abriendo nuevas vías para aplicaciones interesantes. Además que los métodos deben ser de gran importancia para guiar la fabricación de superficies súper hidrófobas para aplicaciones prácticas tales como anti-hielo, autolimpieza y anti-incrustante [8].

\section{PARTE EXPERIMENTAL}




\subsection{MATERIALES}

Se utilizó caolín mineral $\left[\mathrm{Al}_{2} \mathrm{Si}_{2} \mathrm{O}_{5}(\mathrm{OH})_{4}\right]$ pureza $98.6 \%$ exportada por Xinxiang Dongzhenpara, con el fin de reforzar el composito. El ácido esteárico $\left[\mathrm{CH}_{3}\left(\mathrm{CH}_{2}\right)_{16} \mathrm{COOH}\right]$ pureza $99.9 \%$ Croda, se utilizó para la modificación superficial del caolín. El etanol absoluto $\left[\mathrm{C}_{2} \mathrm{H}_{6} \mathrm{O}\right]$ pureza $100 \%$ FERMONT, implementado para modificar al ácido esteárico. El Tetrahidrofurano $\left[\mathrm{C}_{4} \mathrm{H}_{8} \mathrm{O}\right.$ ] (THF) pureza 98.8\% SIGMAALDRICH, utilizado para disolver al PVC. Por último se recicló el policloruro de vinilo $\left[\left(\mathrm{C}_{2} \mathrm{H}_{3} \mathrm{Cl}\right)_{n}\right](\mathrm{PVC})$ por medio de trituración, el cual primero se sometió a un proceso de depuración con el fin de eliminar las impurezas en la superficie y utilizarlo para recubrimiento.

\subsection{MODIFICACIÓN SUPERFICIAL DE CAOLÍN}

Se disolvió ácido esteárico en etanol anhidro, en una proporción de 1:5, respectivamente; a $40^{\circ} \mathrm{C}$ durante 20 minutos, después se adicionaron $5.0 \mathrm{~g}$ de nanopartículas de caolín modificadas [9], las mismas se dispersaron por medio de un baño ultrasónico durante 20 minutos, lo obtenido se sometió a una agitación magnética a $106^{\circ} \mathrm{C}$ durante 2 horas. Después se procedió a realizar un filtrado con el motivo de obtener las nanopartículas modificadas. Finalmente, se llevó a un secado a $120^{\circ} \mathrm{C}$ durante 2 horas con el fin de eliminar el agua ocluida y así obtener las nanopartículas de caolín súper hidrofóbicas.

\subsection{OBTENCIÓN DEL RECUBRIMIENTO}

Se disolvió el polímero (PVC) en (THF) a $30^{\circ} \mathrm{C}$ en agitación magnética durante 20 minutos, sin relación molar, pasado el tiempo se agregaron las nanopartículas de caolín modificado en la disolución obtenida, la solución resultante, se dispersó mediante un baño ultrasónico con un tiempo de 20 minutos. Finalmente se obtuvo una solución homogénea la cual contiene el composito, el cual se procedió a depositar en el Robot Cartesiano con deposición por spray (técnica novedosa), para la obtención del composito con aplicación como un recubrimiento.

\subsection{CARACTERIZACIÓN Y MÉTODOS}

Las medidas del ángulo de contacto del recubrimiento se determinaron con métodos de aproximación, los empleados fueron aumento de imagen y reflexión (recordar que son métodos empíricos). La presciencia de grupos funcionales del composito; estos se presentan a ciertas longitudes de onda, por ende se analizó con un espectrofotómetro infrarrojo de transformada de Fourier (FT-IR) para determinar si están presentes.

\section{RESULTADOS}

Todos los resultados han arrogado que la modificación superficial del caolín es una excelente vía para obtener nanopartículas superhidrofóbicas, esto se comprobó al agregar la disolución en un sustrato de vidrio. Lo acontecido está sustentado en estudios previos sobre aplicaciones de recubrimientos [10]. Se procedió hacer un análisis a las nanopartículas superhidrofóbicas, estas presentaron excelente repelencia hacia el agua, se logró mediante la adición de gotas de agua, las cual se desplazaron rápidamente al contacto con la superficie. Para demostrar su veracidad, se recurrieron los estudios de 
Young [9-11]; presentando los intervalos que determino para clasificar e identificar los tipos materiales existentes. Tabla 1. incluye los valores relevantes a considerar.

Tabla 1. Intervalos del ángulo de contacto de acuerdo a los estudios de Young sobre las superficies sólidas.

\begin{tabular}{|c|c|}
\hline Ángulo & Superficie \\
\hline Menor $\mathrm{o}=15^{\circ}$ & Súper hidrofílico \\
\hline Menor $<0=90^{\circ}$ & Hidrofílico \\
\hline Mayor $>90^{\circ}$, Menor $<150^{\circ}$ & Hidrofóbico \\
\hline Mayor $>150^{\circ}$ & Súper hidrofóbico \\
\hline
\end{tabular}

A continuación en la Figura 1.se presenta el resultado del análisis realizado a las nanopartículas de caolín superhidrofóbicas, demostrando exitosamente que tiene la propiedad de no-mojabilidad; se puede observar que la gota de agua es repelida al momento del contacto con la superficie de las mismas.

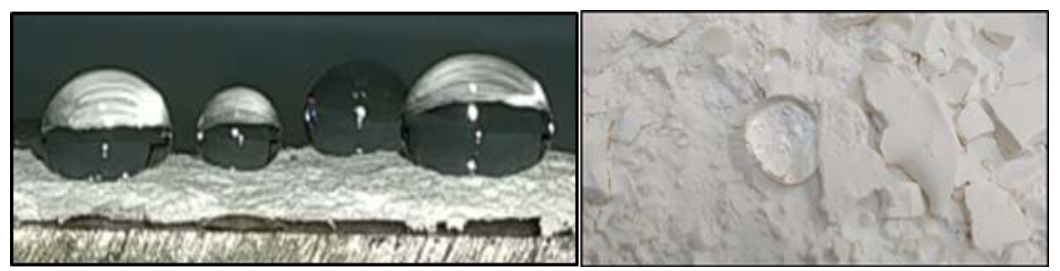

Figura 1. Caolín funcionalizado con ácido esteárico, teniendo un ángulo de contacto entre los $150^{\circ}$ a $157^{\circ}$ aproximados.

Las nanopartículas de caolín antes y después de la modificación química se analizaron por medio de Espectroscopia Infrarroja FTIR, con el objetivo de comprobar la aparición de los grupos funcionales del acido esteárico. En la Figura 2 se aprecia el espectro obtenido por la caracterización FTIR; En a) las bandas características de 3690 y 3624 $\mathrm{cm}^{-1}$ corresponden a la vibración de estiramiento de los grupos hidroxilo $(\mathrm{OH})$ de la caolín [12]. La banda en $1032 \mathrm{~cm}^{-1}$ son de los enlaces Si-O [12-13]. Además, la banda ubicada a $915 \mathrm{~cm}^{-1}$ atribuida a la vibración del estiramiento del Al-OH [13], y las bandas de 791, 755, 683 y $534 \mathrm{~cm}^{-1}$ surgen de las vibraciones del estiramiento de Al-O-Si [1213]. Después de la modificación con ácido esteárico en b) se apreciaron nuevas bandas de absorción a 2920 y $2850 \mathrm{~cm}^{-1}$ (estiramiento asimétrico y simétrico de los grupos ($\mathrm{CH})$ ) [14]; indica que el caolín se funcionalizo adecuadamente con ácido esteárico. 


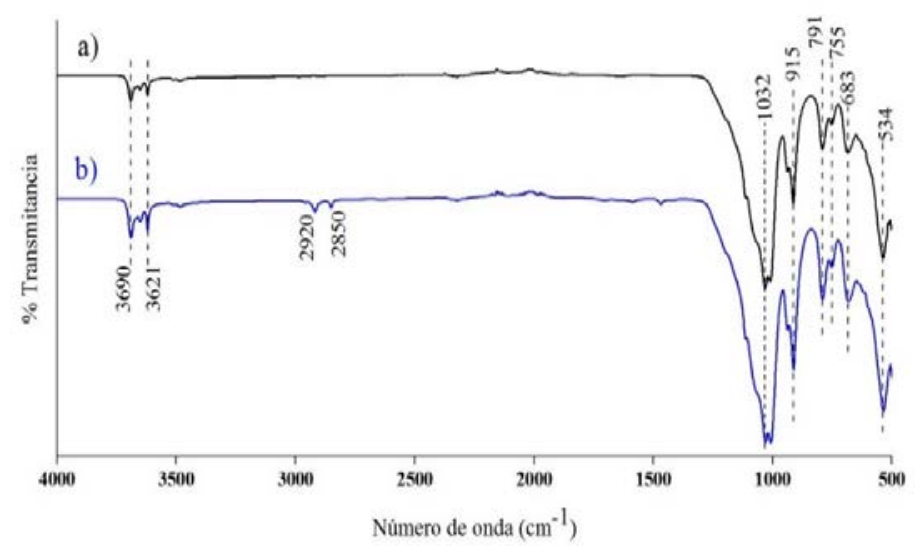

Figura 2. FTIR de los correspondientes polvos, a) Caolín y b) Caolín / ácido esteárico.

Se procedió a analizar nuestra matriz polimérica el Policloruro de vinilo (PVC) reciclado fuera totalmente factible para el composito, primero se disolvió solamente el polímero, por ende se depositó con el robot cartesiano con deposición por spray, ya obteniendo el recubrimiento se llevó a un análisis por métodos de aproximación Figura 3. Obteniendo ángulos mayores a $90^{\circ}$ y menores a $150^{\circ}$, por lo que se presume haber obtenido un recubrimiento hidrofóbico de PVC como se muestra en la Figura 4.

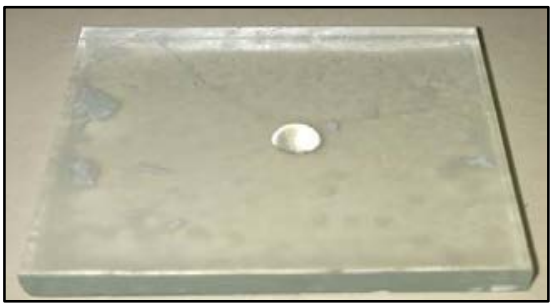

Figura 3. Recubrimiento de (THF-PVC).

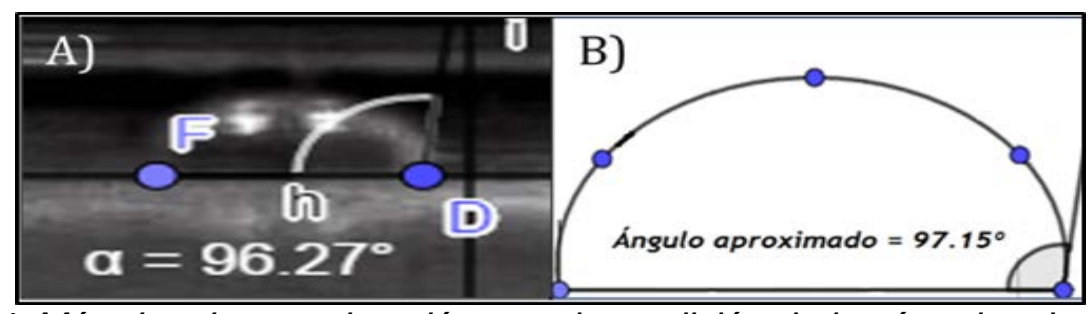

Figura 4. Métodos de aproximación para la medición de los ángulos de contacto

A) Ampliación A.C. Aumento $=96.27^{\circ}$,

B) Reflexión A.C. Reflexión. $=97.15^{\circ}$.

Para el recubrimiento obtenido se realizó un FT-IR con el fin de corroborar la presencia del grupo funcional del monómero $\mathrm{C}$ - $\mathrm{Cl}$, se muestra el espectro obtenido en la Figura 5, donde se aprecia el espectro con bandas de absorción en $612 \mathrm{~cm}^{-1}, 1250 \mathrm{~cm}^{-1}, 1422 \mathrm{~cm}^{-}$ $1,2323 \mathrm{~cm}^{-1}$ y $2977 \mathrm{~cm}^{-1}$ pertenecientes a la vibración de $\mathrm{C}-\mathrm{H}$; y la banda $875 \mathrm{~cm}^{-1}$ pertenece a la vibración de estiramiento de $\mathrm{C}-\mathrm{Cl}$, que muestra la naturaleza del monómero $\left[\mathrm{C}_{2} \mathrm{H}_{3} \mathrm{Cl}\right]$ en el polímero, demostrando que el polímero no sufre modificaciones 


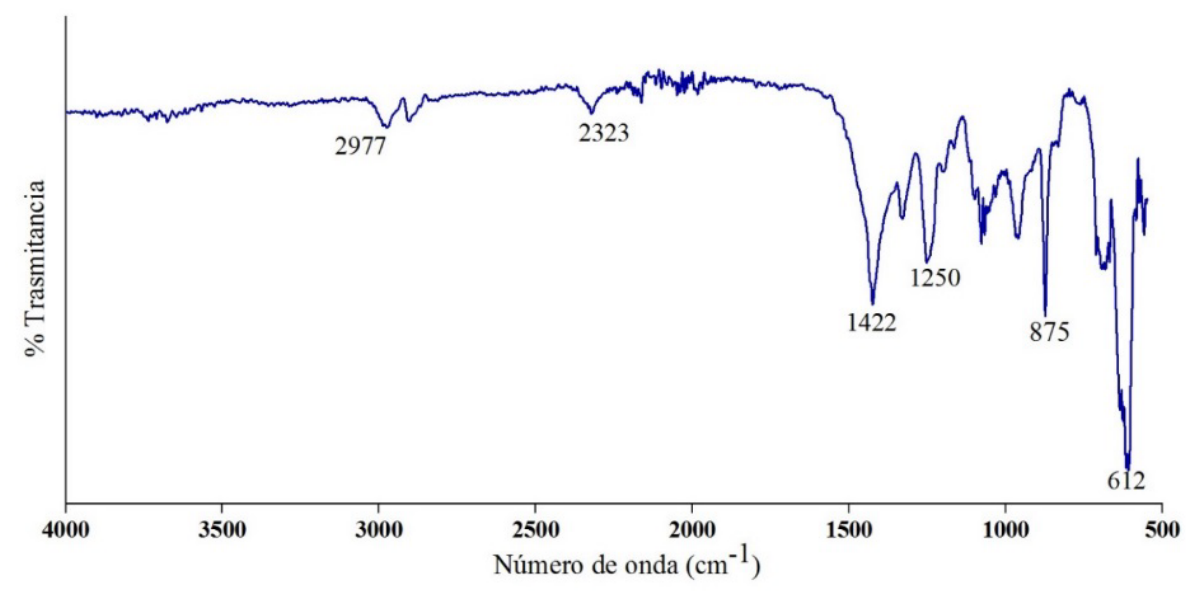

Figura 5. FT-IR del recubrimiento [PVC-THF].

La solución se depositó un sustrato de acero (AISI 304) con técnica novedosa (Robot Cartesiano con deposición por spray), primero se analizó que el composito tuviera la propiedad deseada, de mismo modo se aplicaron gotas de agua como se muestra en la Fig. 6, exitosamente se muestra su gran repelencia al contacto con el líquido.

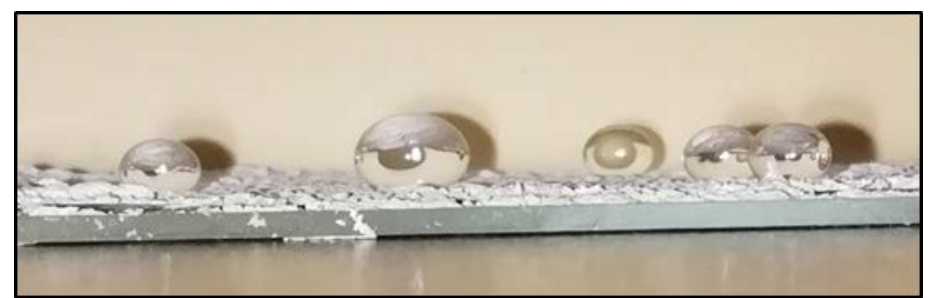

Figura 6 Recubrimiento de (PVC - Caolín superhidrofóbico).

Para proceder con la prueba de corrosión, era relevante primero conocer que los grupos funcionales de los materiales siguieran presentes en el composito, por eso se realizó un FT-IR; el espectro obtenido se muestra en la Figura $7 \mathrm{a}$ y $7 \mathrm{~b}$, se aprecia el espectro correspondiente al [PVC-Caolín] y [Caolín] con la finalidad de hacer una comparativa, con el interés de conocer si siguen apareciendo los grupos funcionales. Se observa que en efecto el polímero no hace que se pierdan las bandas características del ácido esteárico $(\mathrm{COOH})$, cabe resaltar que las bandas características del caolín siguen intactas solo habiendo pequeños desplazamientos. 


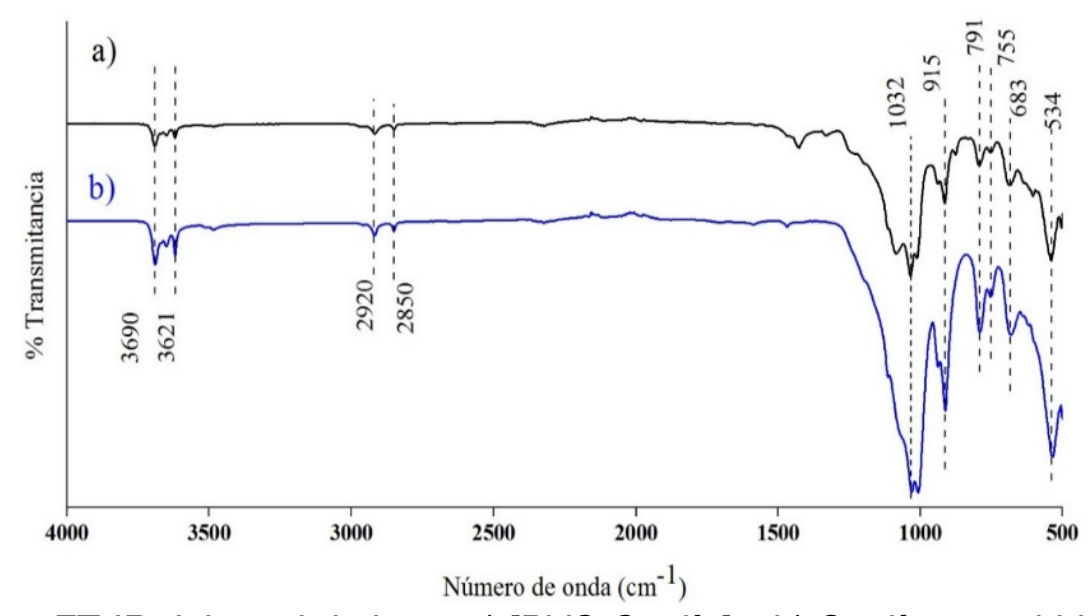

Figura 7. FT-IR del recubrimiento a) [PVC-Caolín] y b) Caolín superhidrofóbico.

De mismo modo se aplicaron los métodos de medición del ángulo de contacto tales como se muestran en la Figura 8 que anteriormente ya se habían presentado Figura 4. Los resultados obtenidos fueron realmente relevantes puesto que arrogaron valores superiores a $150^{\circ}$, mostrando resultados favorables como lo es obtención de la propiedad superhidrofóbica en composito. Después de comprobar la obtención de la propiedad primordial se procedió a realizar las pruebas de corrosión, esto se logró mediante el montaje de una cámara que simulaba una atmosfera corrosiva.

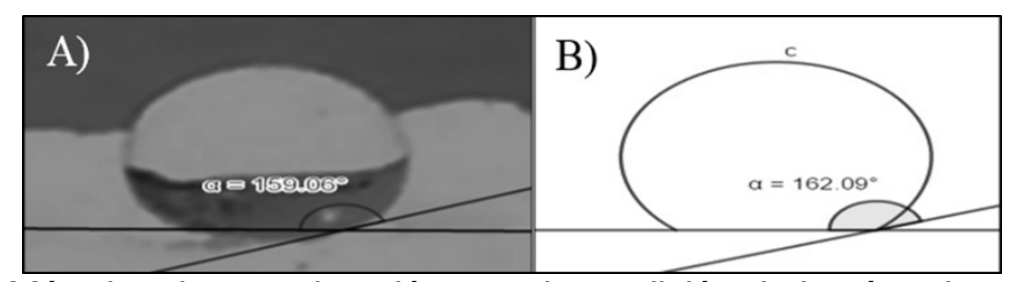

Figura 8. Métodos de aproximación para la medición de los ángulos de contacto

A) Ampliación A.C. Aumento $=159.06^{\circ}$

B) Reflexión A.C. Reflexión $=162.09^{\circ}$

El composito se depositó en los sustratos de aceros (AISI 304) como fueron sometidos a la prueba de corrosión Figura 9, con una duración de 240 horas en una solución altamente corrosiva con el fin de demostrar la eficacia del recubrimiento. Después se procedió a hacer un análisis de corrosión para determinar la eficacia del recubrimiento.

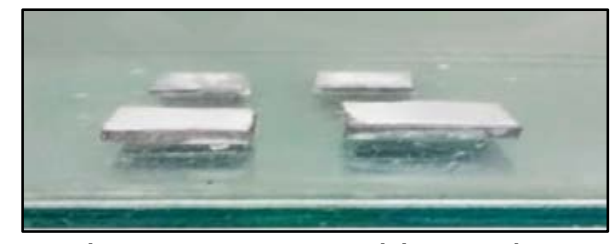

Figura 9. Sustratos de aceros sumergidos en la prueba de corrosión.

Se demostró su efectividad mediante una comparación de pesos de los sustratos antes y después de la prueba de corrosión, el fenómeno de corrosión ataca superficialmente al acero [4]. En este caso se analizaron dos sustratos como se observa en la Figura 10 
(AK01) sin el recubrimiento y (AK03) con el recubrimiento; Los resultados de la prueba de corrosión son verdaderamente exitosos, que muestran (AK03) solo presenta mínimos indicios de corrosión a comparación (AK01) que sufrió un mayor corrosión. Por último se presentan en la Tabla 2, los resultados de pesos demostrando que el composito tiene gran resistencia hacia dicho fenómeno además que no tiene una pérdida significativa de material e incluso vidriando mayores ventajas, con las pruebas obtenidas podemos presumir el composito nanoestructurado es una excelente vía para aplicarse como recubrimiento.

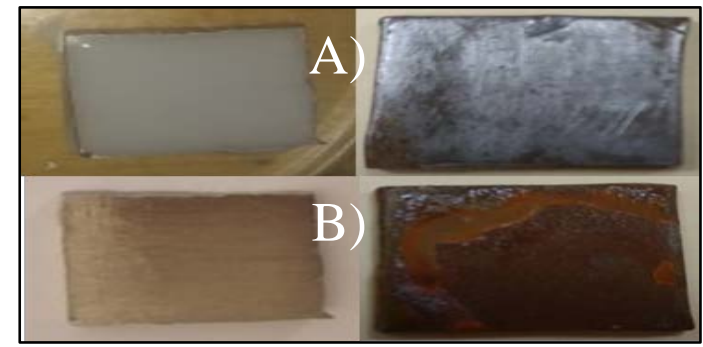

Figura 10. A) AK03 y B) AK01 antes - después de la prueba de corrosión.

Tabla 2. Resultados de pesos antes y después de someterlos a atmosfera corrosiva.

\begin{tabular}{|c|c|c|c|c|}
\hline \multicolumn{5}{|c|}{ Resultados de corrosión (240 horas) } \\
\hline AISI 304 & Peso acero & Antes & Después & Perdida $^{*}$ \\
\hline A) AK03 & $5.6063 \mathrm{~g}$ & $5.6574 \mathrm{~g}$ & $5.5995 \mathrm{~g}$ & $0.0579 \mathrm{~g}$ \\
\hline B) AK01 & $4.8524 \mathrm{~g}$ & $4.8524 \mathrm{~g}$ & $4.5852 \mathrm{~g}$ & $0.2672 \mathrm{~g}$ \\
\hline
\end{tabular}

\section{CONCLUSIONES}

Se ha obtenido un material composito nanoestructurado a base de nanopartículas de caolín - PVC reciclado mediante una síntesis de accesible reproducción, se ha podido depositar con la aplicación de un Robot Cartesiano con deposición por spray (Técnica novedosa) el mismo no representa un precio elevado, el composito presenta propiedades superhidrofóbicas, de acuerdo con los resultados obtenidos de los análisis empleados para medir el ángulo de contacto siendo superiores a los $150^{\circ}$ con una alta repelencia el agua. Mencionando que con esta propiedad adquirida puede retrasar o mitigar el proceso de corrosión, por medio de las pruebas de corrosión se comprobó que también posee la propiedad de resistencia a la corrosión, esto se sustenta con los resultados ya mencionados; cabe resaltar que el composito no sufrió un gran deterioro en su superficie indicando que es una excelente aplicación, lo más relevante demostrando que los polímeros comerciales pueden tener una segunda vida útil otorgando propiedades muy interesantes y benéficas para su aplicación; Esto abre las puertas para nuevas alternativas para la obtención de recubrimientos con mayores propiedades y lo más importante que no generan gran daño al medio ambiente.

\section{AGRADECIMIENTOS}

El autor expresa sus más sinceros agradecimientos a:

Maestra en Nanotecnología Mónica Araceli Camacho González, quien otorgó conocimiento y experiencia; se agradece su participación como asesora que fue muy relevante para el análisis y evaluación general de la investigación. 
Ingeniero en Comunicaciones y Electrónica María del Consuelo Márquez Rueda, por su participación como asesora que fue indispensable para el análisis del alcance, análisis y evaluación de la realización de la investigación.

\section{BIBLIOGRAFÍA}

[1] A. Lafuma, Elsevier, 345 (2013) 457-60.

[2] D. Landolt, Advances in Colloid, 145 (2013) 367-74.

[3] E. Kosmas, Elsevier, 543 (2017) 132-57.

[4] L. Feng, S. Jiang, D. Zhu, Elsevier, 321 (2013) 1857-60.

[5] J. Serrano, J. Alanis, Elsevier, 124 (2017) 153-58.

[6] B. Toshev, D. Platikanov, Colloids and Surfaces, 95 (2006) 177-80.

[7] Z. Tianyi, J. Lei, Colloids and Surfaces, 649 (2018) 324-30.

[8] Z. Mingqian, F. Shile, W. Lei, Elsevier, 647 (2016) 1235-243.

[9] Q. Mengnan, Applied Surface Science, 728 (2017) 299-307.

[10] S. Olavi, S. Pehkonen, Interface Science and Technology, 592 (2016) 133-84.

[11] T. Young, Phil. Trans, 234 (2005) 65-87.

[12] C. Bich, Applied Surface Science, 374 (2005) 1238-247.

[13] V. Farmer, Applied Surface Science, 156 (2007) 134-45.

[14] N. Yang, L. Jicheng, El Sevier, 294 (2017) 101-10. 